\title{
Exchange coupling between magnetic layers across non-magnetic superlattices
}

\author{
M. S. Ferreira \\ Department of Mathematics, Imperial College, London, SW7 2BZ, UK
}

(March 29, 2018)

\begin{abstract}
The oscillation periods of the interlayer exchange coupling are investigated when two magnetic layers are separated by a metallic superlattice of two distinct non-magnetic materials. In spite of the conventional behaviour of the coupling as a function of the spacer thickness, new periods arise when the coupling is looked upon as a function of the number of cells of the superlattice. The new periodicity results from the deformation of the corresponding Fermi surface, which is explicitly related to a few controllable parameters, allowing the oscillation periods to be tuned.
\end{abstract}

PACS numbers: 75.30.Et, 75.70.-i, 75.50.Rr

\section{INTRODUCTION}

Oscillatory interlayer exchange coupling in metallic magnetic multilayers causes the magnetizations of neighbouring magnetic layers separated by non-magnetic spacers to be spontaneously aligned ferromagnetically or antiferromagnetically depending on the thickness of the spacer material. This phenomenon has been intensively studied both experimentally and theoretically over the last few yearst 2 .

The relation between the periods, by far the most investigated feature of the oscillations, and the electronic structure of multilayered systems is a fundamental aspect of the mechanism responsible for the origin of this phenomenon. In fact, the periods were originally shown to be dependent on the shape of the spacer Fermi surface, where two distinct

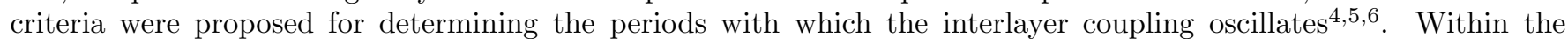
quantum well theory the wave vectors yielding the effective oscillation periods correspond to extremal radii of the Fermi surface in the direction perpendicular to the layers, or in other words to half the caliper measurementst. On the other hand, in the RKKY theory adapted to the layered geometry, the periods are associated with wave vectors perpendicular to the layers linking two points of the Fermi surface with antiparallel velocities 5 . 6 . Despite the distinction between those geometrical criteria, the periods predicted by both theories, at least in their original formulations, agree in most cases. When they do not coincide, such as, for example, when the lattice lacks reflection symmetry about the layer planes, suitable transformations of the Fermi surface can be performed, which reestablish the correspondence between the quantum well and the RKKY periods. Recently howerer fundamental oscillation periods not directly associated with the shape of the spacer Fermi surface were obtained 8 9, 10. Those periods, which correspond to higher order contributions to the exchange coupling, are no longer determined by the above geometrical criteria but by a set of more general conditions on the electronic structure of the system, although they are still indirectly dependent on the shape of the Fermi surface. These conditions provide a systematic way of determining the oscillation periods involved in the interlayer coupling.

In order to test the applicability of those general conditions, it is worth investigating them in situations where the spacer Fermi surface, clearly a relevant factor in this matter, is variable. One approach is to use an alloy spacer of variable composition although the Fermi surface is no longer sharply defined. Also, it is well know that the scattering of electrons by random impurities strongly damps the oscillations. In fact, the amplitude of the coupling is found to decay exponentially with the concentration of impurities 11 . 12 . An alternative to an alloy is a metallic superlattice whose Fermi surface depends on the proportions and layer thicknesses of the constituent materials but where the oscillations are not damped by incoherent electronic scattering. Moreover, nature provides us with only a limited number of non-magnetic metals. However, by combining distinct materials in superlattices the number of possible non-magnetic superlattices is infinite.

The purpose of this communication is to investigate the oscillation periods of the interlayer exchange coupling when the spacer is a non-magnetic superlattice. It is shown that the alteration of the oscillation periods reflects the deformation of the corresponding Fermi surface only when the coupling is investigated as a function of the number of superlattice cells separating the magnetic layers. In the simple model treated here, the oscillation periods of the coupling are written in terms of controllable parameters which are related to the composition of the superlattices, and which, accordingly, allow the periods to be tuned. It is hoped that such a simple model reflects what occurs in realistic systems, and this may be confirmed by future experiments. 
The sequence adopted in this work is described as follows. In order to make the work self-contained, a brief summary of the general method for determining the oscillation periods in a multi-orbital tight-binding model is presented in section II. Secondly, the method is shown to be applicable when the spacer is a superlattice, using the clear correspondence between orbital and intracell indices. Subsequently, the oscillation periods are obtained following the analysis of the electronic structure of the system and are explicitly written in terms of parameters associated with the superlattice composition.

\section{OSCILLATION PERIODS}

A brief summarypf the general method for determining the oscillation periods of the interlayer coupling is presented here. See reference 9 for a comprehensive discussion of the method.

For the sake of simplicity, we consider two parallel magnetic planes, labelled 0 and $n$, embedded in an infinite non-magnetic metal. As far as the coupling as a function of the spacer thickness is concerned, the number of magnetic planes influences only the phase and amplitude of the oscillations and does not affect the periods 13 . Since the periods are the main concern here, the restriction to two single magnetic planes does not pose any limitations on the results obtained.

The interlayer coupling $\mathcal{J}$ is given by the difference in total energy between the antiferromagnetic and ferromagnetic configurations or, in other words, by the energy necessary to flip the magnetization in one of the planes. Based on the formalism introduced by d'Albuquerque e Castro et al, the coupling $\mathcal{J}$ is written in terms of the one-electron Green functions of the multilayered system by the following expression

$$
\mathcal{J}=\sum_{\mathbf{q}_{\|}} \int d \omega \mathcal{F}\left(\mathbf{q}_{\|}, \omega\right)
$$

where $\mathcal{F}\left(\mathbf{q}_{\|}, \omega\right)=f(\omega) \operatorname{Im} \operatorname{Tr} \ln \left\{1+4 G_{n 0}^{\uparrow}\left(\mathbf{q}_{\|}, \omega\right) V_{e x} G_{0 n}^{\downarrow}\left(\mathbf{q}_{\|}, \omega\right) V_{e x}\right\}$. In the equations above $G_{n 0}^{\sigma}\left(\mathbf{q}_{\|}, \omega\right)$ is the propagator between planes 0 and $n$ for electrons with spin $\sigma$ in the ferromagnetic configuration of the system, $f(\omega)$ is the Fermi function, $V_{e x}$ is a diagonal matrix in orbital indices representing the strength of the local exchange potential in the ferromagnetic layers, and the sum over $\mathbf{q}_{\|}$is restricted to the two-dimensional Brillouin zone. Bearing in mind that the exchange splitting of the magnetic material is localized on the two planes 0 and $n$, the propagators $G$ can be rewritten as a function of the bulk spacer propagators $g_{n 0}$ and $g_{0 n}$, i.e.,

$$
G_{n 0}^{\sigma}\left(\mathbf{q}_{\|}, \omega\right)=G^{\sigma}\left(g_{n 0}\left(\mathbf{q}_{\|}, \omega\right), g_{0 n}\left(\mathbf{q}_{\|}, \omega\right)\right)
$$

Moreover, it can be shown that within the single-band model the dependence of $G^{\sigma}$ upon those propagators is such that $G_{n 0}^{\sigma}=G^{\sigma}\left(g_{n 0} g_{0 n}\right)$. It is clear that the spacer thickness dependence of the interlayer coupling is entirely determined by the off-diagonal propagators $g_{n 0}$ and $g_{0 n}$. The investigation of the oscillation periods thus requires an analysis of $g_{n 0}$ as a function of the interplane distance.

In the layered geometry, where $\mathbf{q}_{\|}$represents the in-plane wave-vector, the expression for a general matrix element $g_{\ell m}^{\mu \nu}$ representing the propagation from an orbital $\mu$ at plane $\ell$ into an orbital $\nu$ at plane $m$ of an electron at the Fermi level $E_{F}$ is given by

$$
g_{\ell m}^{\mu \nu}\left(\mathbf{q}_{\|}, E_{F}\right)=\left(\frac{d}{2 \pi}\right) \sum_{s} \int_{-\frac{\pi}{d}}^{\frac{\pi}{d}} d q_{\perp} \frac{a_{s \mu}^{*}\left(q_{\perp}\right) a_{s \nu}\left(q_{\perp}\right) e^{-i q_{\perp}(\ell-m) d}}{E_{F}-E_{s}\left(\mathbf{q}_{\|}, q_{\perp}\right)+i 0^{+}}
$$

where the integration variable $q_{\perp}$ is the wave vector perpendicular to the layers, $d$ is the interplane distance, $E_{s}\left(\mathbf{q}_{\|}, q_{\perp}\right)$ describes the bulk spacer band structure, $s$ is the band index, and $a_{s \mu}\left(q_{\perp}\right) \equiv\left\langle q_{\perp} s \mid q_{\perp} \mu\right\rangle$ is the projection of the eigenstate $s$ into the orbital $\mu$ for a given $q_{\perp}$. By changing the integration contour from the real axis to the boundaries of a semi-infinite rectangle in the complex plane whose base lies on the real axis between $-\pi / d$ and $\pi / d$, the evaluation of the integral above is simplified to the determination of the poles of the integrand inside the appropriate contour and their respective residues. The rectangle is in the upper-half plane for $\ell<m$ and in the lower-half plane otherwise. Clearly, the poles are given by the values of $q_{\perp}$ satisfying the condition $E_{F}{ }^{+}-E_{s}\left(\mathbf{q}_{\|}, q_{\perp}\right)=0$, where $E_{F}{ }^{+}=E_{F}+i 0^{+}$.

By adding the residues associated with the contributory poles, labelled $q_{j}^{s}$, an analytical expression for $g_{\ell m}$ is obtained, i.e.,

$$
g_{\ell m}\left(\mathbf{q}_{\|}, \omega\right)=\sum_{s} \sum_{j} A_{s j} e^{-i q_{j}^{s}(\ell-m) d}
$$


where the matrix elements of the matrix $A_{s j}$ are

$$
A_{s j}^{\mu \nu}\left(\mathbf{q}_{\|}, \omega\right)=-i d a_{s \mu}^{*}\left(q_{j}^{s}\right) a_{s \nu}\left(q_{j}^{s}\right)\left\{\left[\frac{\partial E_{s}\left(\mathbf{q}_{\|}, q_{\perp}\right)}{\partial q_{\perp}}\right]_{q_{\perp}=q_{j}^{s}}\right\}^{-1} .
$$

The coefficients $A_{s j}^{\mu \nu}$ depend neither on $\ell$ nor on $m$ and the only dependence on the distance between the planes is inside the argument of the exponentials. It should be stressed that this simple method for obtaining the one-electron propagators is exclusive to the one-dimensional case, and therefore for fixed values of $\mathbf{q}_{\|}$. The exponentials in Eq. (4) are independent of the orbital indices, which means that all matrix elements oscillate with the same periods. Note that in calculating Eq.(5) it was assumed that the coefficients $a_{s \mu}\left(q_{\perp}\right)$ have neither singularities nor branch points inside the integration contour. In this way, the coefficients are evaluated just at the poles $q_{j}^{s}$. In general, branch cuts associated with the coefficients $a_{s \mu}\left(q_{\perp}\right)$ may exist, but because they may be chosen not to intersect the real axis, the factor $e^{-i q_{\perp}(\ell-m) d}$ in the integrand of Eq.(3) ensures that the corresponding contribution is negligible for large $|\ell-m|$. Therefore, the expression above is correct in the asymptotic limit of large interplane distanceg.

It is clear from Eq.(伍) that $g_{\ell m}$ is an oscillatory function of the distance between the planes and it oscillates with different periods, each one associated with its respective wave vector $q_{j}^{s}$. For this reason it is interesting to look at the physical significance of the poles. The poles may be real or complex, but we note that only the real poles contribute significantly to the coupling. This is because complex poles, when inserted in Eq.(1), damp the oscillations and are important only in cases of very thin spacers. Real poles, which are obtained from the electronic structure of the bulk spacer, are just the perpendicular coordinates of the Fermi surface for a fixed value of $\mathbf{q}_{\|}$. In other words, they are the perpendicular components of the wave vectors with which electrons of energy $E_{F}$ propagate across the spacer.

Having investigated $g_{\ell m}$ as a function of the interplane distance, it is worth stressing that the functions $\mathcal{F}$ must oscillate with the sime periods. When expanded in a Fourier series, taking into account the possibility of quasiperiodicity of $g_{n 0}$ 迎 13 , the function $\mathcal{F}$ is written as

$$
\mathcal{F}=\sum_{\left(m_{1}^{1}, \ldots, m_{j}^{s}, \ldots\right)} \mathcal{C}_{\left(m_{1}^{1}, \ldots, m_{j}^{s}, \ldots\right)} e^{i n d \sum_{s j}\left(m_{j}^{s} q_{j}^{s}\right)},
$$

where $\mathcal{C}_{\left(m_{1}^{1}, \ldots, m_{j}^{s}, \ldots\right)}$ are the Fourier coefficients determining the amplitude of the oscillations and depend on the exact form of the function $\mathcal{F}$. The indices $\left(m_{1}^{1}, \ldots, m_{j}^{s}, \ldots\right)$ are integers and there are as many as the number of poles $q_{j}^{s}$. Note that the periods are given by $\lambda_{\left(m_{1}^{1}, \ldots, m_{j}^{s}, \ldots\right)}=2 \pi /\left(\sum_{s j} m_{j}^{s} q_{j}^{s}\right)$ and do not depend on the exact form of $\mathcal{F}$, since they are obtained from the bulk spacer band structure. Because the indices $m_{j}^{s}$ run over the set of integers, there is always an infinite number of periods defined by Eq.(6), even for a small number of poles $q_{j}^{s}$. In spite of the somewhat congested notation of the general expression above, in practice there are only a few indices involved due to the restricted number of real solutions crossing the Fermi level. In fact, the number of solutions is related to the number of sheets of the Fermi surface.

When Eq.(6) is inserted into Eq.(11), the two-dimensional integral can $_{\text {an }}$ be evaluated through the stationary phase approximation, valid in the asymptotic limit of large spacer thickness 1 . 5 . In doing so, the periods of the coupling are identified by selecting the periods of $\mathcal{F}$ associated with wave vectors satisfying the following condition,

$$
\sum_{s j}\left[m_{j}^{s} \nabla_{\|} q_{j}^{s}\left(\mathbf{q}_{\|}, E_{F}\right)\right]=0
$$

where $\nabla_{\|}$is the two-dimensional gradient in $\mathbf{q}_{\|}$-space. The equation above gives the necessary conditions for constructive interference between electrons across the spacer. The otherwise destructive interference does not contribute to the coupling. Eq.(7) simply selects the points across the two-dimensional Brillouin zone which yield the effective periods with which the couplipg oscillates.

As pointed out in reference 9 , although the wave vectors $q_{j}^{s}$ are directly related to the Fermi surface, the general wave vectors $\sum_{s j}\left(m_{j}^{s} q_{j}^{s}\right)$ are not. This is a result of the interference between incommensurate wave vectors $q_{j}^{s}$ giving rise to a new set of periods not directly obtained from the structure of the Fermi surface. Consequently, geometrical criteria cannot be used in general to select the oscillation periods. However, for Fermi surfaces with a single sheet, where the off-diagonal propagators oscillate as a function of the interplane distancewith only one period, the selection rules given by Eq.(7) coincide with the geometrical criteria of the RKKY theory is that Eq.(7) determines the respective harmonics, in addition to the fundamental RKKY periods.

In summary, the determination of the oscillation periods results from the analysis of the bulk spacer off-diagonal propagators where the relevant real wave vectors, under the stationary phase condition, are obtained from the band structure. When dealing with multi-sheet Fermi surfaces, the possibility of interferences between incommensurate wave vectors may introduce new features in the oscillations, which are not present in the case of single-period oscillations of the propagators. 


\section{SUPERLATTICED SPACERS}

Having summarized the method for determining the oscillation periods of the coupling, it is worth stressing that no particular form for the spacer material was assumed. The only information required is the bulk band structure which makes possible the investigation of the coupling across arbitrary spacers. Spacers consisting of superlattices of distinct non-magnetic materials will be investigated here. More specifically, the material into which the magnetic planes are embedded is composed of two non-magnetic metals $A$ and $B$ with respective thicknesses $N_{A}$ and $N_{B}$ periodically alternated, as schematically displayed in figure 1. In order to make a distinction, these structures will be hereafter named superlatticed spacers, in contrast to the conventional single-layered spacers. The interlayer coupling across superlatticed spacers can be looked upon as a function of different thicknesses, namely $N_{A}, N_{B}, N$ and $N_{c}$, where $N_{c}$ represents the number of cells separating the magnetic planes. Each cell is formed by a set of $N_{A}$ planes of metal $A$ and $N_{B}$ planes of metal $B$. As far as the oscillation periods are concerned, the coupling across superlatticed spacers as a function of $N_{A}$ or $N_{B}$ does not present new features when compared to the coupling across conventional singlelayered spacers as a function of $N_{A}$ or $N_{B}$, respectively. In other words, if a single-layered spacer of material $A$ yields an interlayer coupling with period $\lambda_{A}$ and likewise $\lambda_{B}$ is the period for the coupling across a single-layered spacer of material $B$, the coupling across a superlatticed spacer of materials $A$ and $B$ will oscillate with period $\lambda_{A}$ when seen as a function of $N_{A}$ and with period $\lambda_{B}$ when seen as a function of $N_{B}$. However, when looked upon as a function of $N_{c}$, for fixed values of $N_{A}$ and $N_{B}$, the coupling oscillates with periods different from $\lambda_{A}$ and $\lambda_{B}$. Although the superlattice Fermi surface is different from the individual Fermi surfaces of materials A and B separately, it is only when investigated as a function of $N_{c}$ that the deformation of the Fermi surface is translated into a change in the periods.

Following the steps of the previous section, the bulk spacer band structure must be calculated. For the sake of simplicity, the single-band tight-binding model is considered assuming a simple cubic lattice structure for the superlattice grown along the (100) direction. For the two given materials $A$ and $B$, the in-plane energies are labelled $\epsilon_{A}$ and $\epsilon_{B}$, and the interplane hoppings are $t_{A}$ and $t_{B}$. The hopping between planes $A$ and $B$ is represented by $t_{A B}$. We recall that the tight-binding parameters above depend on the in-plane wave vector $\mathbf{q}_{\|}$through $\epsilon_{\beta}\left(\mathbf{q}_{\|}\right)=$ $\epsilon_{\beta}^{0}+2 t_{\beta}^{0}\left[\cos \left(q_{x} d\right)+\cos \left(q_{y} d\right)\right]$ and $t_{\beta}\left(\mathbf{q}_{\|}\right)=t_{\beta}^{0}$, where $\beta$ is either $A$ or $B, \epsilon_{\beta}^{0}$ is the on-site energy and $t_{\beta}^{0}$ is the hopping between nearest-neighbour sites in material $\beta$. Note that $q_{z}$ is perpendicular to the layers whereas $q_{x}$ and $q_{y}$ are the coordinates of $\mathbf{q}_{\|}$.

Because each set of $N$ planes is periodically repeated, where $N=N_{A}+N_{B}$, the system can be represented by cells of $N$ planes equally spaced. Consequently, the tight-binding Hamiltonian of the infinite multilayered system, when written in the appropriate basis, becomes a $N \times N$ matrix given by

$$
\mathcal{H}=\left(\begin{array}{cc}
{\left[\mathcal{H}_{A}\left(N_{A}\right)\right]} & {\left[\mathcal{H}_{A B}\right]} \\
{\left[\mathcal{H}_{B A}\right]} & {\left[\mathcal{H}_{B}\left(N_{B}\right)\right]}
\end{array}\right)
$$

where $\left[\mathcal{H}_{A}\left(N_{A}\right)\right]$ and $\left[\mathcal{H}_{B}\left(N_{B}\right)\right]$ are square matrices of order $N_{A}$ and $N_{B}$, respectively. The sub-matrices $\left[\mathcal{H}_{A}\left(N_{A}\right)\right]$ and $\left[\mathcal{H}_{B}\left(N_{B}\right)\right]$ are explicitly given by

$$
\left.\left[\mathcal{H}_{\beta}\left(N_{\beta}\right)\right]=\left(\begin{array}{cccc}
\epsilon_{\beta} & t_{\beta} & 0 & 0 \\
t_{\beta} & \epsilon_{\beta} & t_{\beta} & 0 \\
0 & t_{\beta} & \epsilon_{\beta} & t_{\beta} \\
0 & 0 & t_{\beta} & \epsilon_{\beta}
\end{array}\right)\right\} N_{\beta}
$$

Note that $\left[\mathcal{H}_{\beta}\left(N_{\beta}\right)\right]$ is a tri-diagonal matrix equivalent to the single-band tight-binding Hamiltonian of a slab consisting of $N_{\beta}$ planes. The sub-matrices off the diagonal in Eq.(8) are $N_{A} \times N_{B}$ and $N_{B} \times N_{A}$ matrices such that

$$
\left[\mathcal{H}_{A B}\right]=t_{A B} \underbrace{\left(\begin{array}{cccc}
0 & 0 & 0 & e^{-i q_{\perp} N d} \\
0 & 0 & 0 & 0 \\
1 & 0 & 0 & 0
\end{array}\right)}_{N_{B}}\} N_{A}
$$

and $\left[\mathcal{H}_{B A}\right]=\left[\left(\mathcal{H}_{A B}\right)^{\dagger}\right]$. In the matrix directly above $q_{\perp}$ is the wave vector perpendicular to the layers and $d$ is the interplane distance, which is assumed to be the same for both materials. The basis producing such matrices results from a Fourier transform of the localized-atomic-orbital basis along the direction perpendicular to the layers. Since the cells of $N$ planes are equally spaced by $N d$, the associated wave vector $q_{\perp}$ is in the range $\left[\frac{-\pi}{N d}, \frac{\pi}{N d}\right]$, that is, the corresponding Brillouin zone along this direction is reduced by a factor $N$. The tight-binding Hamiltonian above 
is equivalent to the $N$-band Hamiltonian of a one-dimensional system. In other words, the $N$ intracell planes may be considered as orbitals of a linear chain whose nearest neighbour elements are $N d$ apart. Having established this correspondence, hereafter intracell and orbital indices will be mentioned with no distinction between them.

Following section III, the oscillation periods result from the analysis of the bulk spacer off-diagonal propagators as a function of the distance between the planes. Note however that in the case treated here the effective interplane distance must be the distance between the cells, i.e., $D=N d$. Hence, the off-diagonal propagators as a function of the number of cells $N_{c}$ are the ones to be investigated. These propagators depend on the band structure of the system, as clearly shown in Eq.(3). The original tight-binding Hamiltonian is partially diagonalized by both in-plane and perpendicular Fourier transforms, the remaining problem being the determination of the eigenvalues of the $N \times N$ matrix in Eq.(8). Therefore, the information on the oscillation periods of the coupling in a superlatticed system depends on the diagonalization of this matrix, which will be presented in the next section.

To maintain the correspondence between the intracell planes of the superlattice and orbital indices of the linear chain, one has to assume that in the case of superlatticed spacers the magnetic planes embedded in the non-magnetic system must be formed by two separate magnetic cells. However, for the sake of simplicity and with no loss of generality, we consider only two magnetic planes. If we label the magnetic planes with the intracell indices $\alpha$ in the cell 0 and $\alpha^{\prime}$ in the cell $N_{c}$, the relevant Green functions to be calculated are the off-diagonal propagators $g_{0 N_{c}}^{\alpha \alpha^{\prime}}\left(\mathbf{q}_{\|}, \omega\right)$, i.e.,

$$
g_{0 N_{c}}^{\alpha \alpha^{\prime}}\left(\mathbf{q}_{\|}, \omega\right)=\left(\frac{D}{2 \pi}\right) \sum_{s} \int_{-\frac{\pi}{D}}^{\frac{\pi}{D}} d q_{\perp} \frac{a_{s \alpha}^{*}\left(q_{\perp}\right) a_{s \alpha^{\prime}}\left(q_{\perp}\right) e^{i q_{\perp} N_{c} D}}{\omega-E_{s}\left(\mathbf{q}_{\|}, q_{\perp}\right)+i 0^{+}}
$$

In addition to the fact that $\alpha$ and $\alpha^{\prime}$ are intracell indices, the only difference between the equation above and Eq.(3) is the interplane distance $D$ which modifies the integral limits. Similarly to section [I], the integral above is evaluated through the residues method, where the determination of the poles follow the diagonalization of the Hamiltonian.

\section{A. Electronic structure}

Making use of the particular symmetry of the matrices displayed above, the determinant of $\mathcal{H}$ in Eq.(8) can be written in terms of the sizes of its sub-matrices $N_{A}$ and $N_{B}$ yielding a simple analytical expression for the oscillation periods of the one-electron propagators. The energy eigenvalues $\omega$ are solutions of the equation $\operatorname{det}|\mathcal{H}-\omega \hat{\mathbf{1}}|=0$, where $\hat{\mathbf{1}}$ is the identity matrix. In this case the sub-matrices along the diagonal become

$$
\left[\mathcal{H}_{\beta}\left(N_{\beta}\right)-\omega \hat{\mathbf{1}}\right]=\underbrace{\left(\begin{array}{cccc}
\epsilon_{\beta}-\omega & t_{\beta} & 0 & 0 \\
t_{\beta} & \epsilon_{\beta}-\omega & t_{\beta} & 0 \\
0 & t_{\beta} & \epsilon_{\beta}-\omega & t_{\beta} \\
0 & 0 & t_{\beta} & \epsilon_{\beta}-\omega
\end{array}\right)}_{N_{\beta}},
$$

whereas the block matrices off the diagonal are still given by $\left[\mathcal{H}_{A B}\right]$ and $\left[\mathcal{H}_{B A}\right]$.

The determinant of a $N \times N$ square matrix is a sum of $N$ ! terms. Those terms are made of products of $N$ matrix elements, following all their possible permutations. Because the sub-matrices on the diagonal have only two distinct non-zero elements, the evaluation of their determinants by counting the number of combinations allowed by the possible permutations is not particylarly difficult and can be expressed in term of the sizes $N_{\beta}$. The solution of this combinatorial problem is given by 16

$$
\operatorname{det}\left|\mathcal{H}_{\beta}\left(N_{\beta}\right)-\omega \hat{\mathbf{1}}\right|=\sum_{j=0}^{N_{\beta} / 2}(-1)^{j}\left(\begin{array}{c}
N_{\beta}-j \\
j
\end{array}\right)\left(\epsilon_{\beta}-\omega\right)^{N_{\beta}-2 j}\left(t_{\beta}\right)^{2 j},
$$

where $j$ is an integer, the upper limit of the sum is the integer part of $N_{\beta} / 2$ and

$$
\left(\begin{array}{c}
N_{\beta}-j \\
j
\end{array}\right)=\frac{\left(N_{\beta}-j\right) !}{\left(N_{\beta}-2 j\right) ! j !}
$$

is the binomial coefficient. Note that the expression above is valid for symmetric tri-diagonal matrices with similar elements along each of the diagonals. Bearing in mind that $\mathcal{H}_{\beta}\left(N_{\beta}\right)$ is equivalent to the slab Hamiltonian, the values of $\omega$ satisfying $\operatorname{det}\left|\mathcal{H}_{\beta}\left(N_{\beta}\right)-\omega \hat{\mathbf{1}}\right|=0$ through Eq.(13) are just the associated eigenvalues. 
The Hamiltonian $\mathcal{H}$ in Eq.(8) is almost tri-diagonal. The matrix $\mathcal{H}$ is not tri-diagonal due to two of its elements, namely $\mathcal{H}_{1, N}$ and $\mathcal{H}_{N, 1}$, which are non-zero. Therefore, an expression for the determinant of $\mathcal{H}$ is not expected to be very different from the one in Eq.(13). In fact, by considering the only two non-zero elements of the submatrices in Eq.(10) and recounting the number of possible permutations, the determinant of the matrix $\mathcal{H}$ can be expressed in terms of the determinants of the matrices $\mathcal{H}_{A}\left(N_{A}\right)$ and $\mathcal{H}_{B}\left(N_{B}\right)$. In the similar case of $\mathcal{H}-\omega \hat{\mathbf{1}}$, the determinant is given by

$$
\begin{aligned}
\operatorname{det}|\mathcal{H}-\omega \hat{\mathbf{1}}|= & \operatorname{det}\left|\mathcal{H}_{A}\left(N_{A}\right)-\omega \hat{\mathbf{1}}\right| \times \operatorname{det}\left|\mathcal{H}_{B}\left(N_{B}\right)-\omega \hat{\mathbf{1}}\right| \\
& -2\left(t_{A B}\right)^{2} \operatorname{det}\left|\mathcal{H}_{A}\left(N_{A}-1\right)-\omega \hat{\mathbf{1}}\right| \times \operatorname{det}\left|\mathcal{H}_{B}\left(N_{B}-1\right)-\omega \hat{\mathbf{1}}\right| \\
& +\left(t_{A B}\right)^{4} \operatorname{det}\left|\mathcal{H}_{A}\left(N_{A}-2\right)-\omega \hat{\mathbf{1}}\right| \times \operatorname{det}\left|\mathcal{H}_{B}\left(N_{B}-2\right)-\omega \hat{\mathbf{1}}\right| \\
& +\left(t_{A B}\right)^{2}\left(t_{A}\right)^{N_{A}-1}\left(t_{B}\right)^{N_{B}-1} \cos \left(q_{\perp} N d\right) .
\end{aligned}
$$

Note that the equation above is written in terms of lower-order determinants of Eq.(13) and is an analytical expression for $\operatorname{det}|\mathcal{H}-\omega \hat{\mathbf{1}}|$ in terms of its sizes $N_{A}$ and $N_{B}$.

It is instructive to point out that when $A=B$, that is, when a single-layered spacer is considered, the usual eigenvalues $\omega\left(q_{\perp}\right)=\epsilon+2 t \cos \left(q_{\perp} d\right)$ of an infinite linear chain are obtained from Eq. 15 through the condition $\operatorname{det}|\mathcal{H}-\omega \hat{\mathbf{1}}|=0$.

The eigenvalues $\omega$ of the Hamiltonian $\mathcal{H}$ are solutions of the polynomial equation defined by Eqs.(15) and (13) through the condition $\operatorname{det}|\mathcal{H}-\omega \hat{\mathbf{1}}|=0$. To illustrate this point, figure 2 displays the band structure of a superlatticed system consisting of two materials $A$ and $B$ whose tight-binding parameters are arbitrarily chosen as $\epsilon_{A}^{0}=\epsilon_{B}^{0}=0$, $t_{A}=-1 / 2$ and $t_{B}=-1 / 3$. Two cases are shown in figure 2, namely $N=3$ and $N=6$. In each case a number of possible values for $N_{A}$ and $N_{B}$ is presented. Due to the equivalence between the single-band-cellular and multi-band linear Hamiltonians, it is clear in all the cases that, for a given $N_{A}$, there are $N$ solutions per $q_{\perp}$ forming the respective bands. All bands are separated by energy gaps except for the ones in figure 22A corresponding to $N_{A}=0$ and $N_{A}=3$. Those two cases correspond to single materials $B$ and $A$, respectively.

Since we are interested in determining the oscillation periods of the one-electron propagators, what is needed from the polynomial equation defined by Eqs.(15) and (13) is the wave vector $q_{\perp}$ with which electrons with energy $\omega$ propagate across the superlatticed spacer, that is, the poles $q_{\perp}$ of the integrand in Eq.(11). Whereas the determination of the eigenvalues requires finding the zeros of an $N$-th degree polynomial in $\omega$, the calculation of the wave vector $q_{\perp}$ for a given energy $\omega$ is straightforward due to the simple dependence of Eq.(15) on $q_{\perp}$. The advantage of writing the expression of the determinant in terms of the number of planes $N_{A}$ and $N_{B}$ becomes clear, for the oscillation periods can be written in terms of those parameters. Furthermore, bearing in mind that the wave vectors $q_{\perp}$ are the perpendicular components of the spacer Fermi surface, we can describe how the Fermi surface is deformed with the composition of the cells. The wave vector $q_{\perp}$ is such that

$$
\begin{aligned}
\cos \left(q_{\perp} N d\right)= & \frac{-1}{\left(t_{A}\right)^{N_{A}}\left(t_{B}\right)^{N_{B}}}\left\{\operatorname{det}\left|\mathcal{H}_{A}\left(N_{A}\right)-\omega \hat{\mathbf{1}}\right| \times \operatorname{det}\left|\mathcal{H}_{B}\left(N_{B}\right)-\omega \hat{\mathbf{1}}\right|\right. \\
& -2 t_{A} t_{B} \operatorname{det}\left|\mathcal{H}_{A}\left(N_{A}-1\right)-\omega \hat{\mathbf{1}}\right| \times \operatorname{det}\left|\mathcal{H}_{B}\left(N_{B}-1\right)-\omega \hat{\mathbf{1}}\right| \\
& \left.+t_{A}^{2} t_{B}^{2} \operatorname{det}\left|\mathcal{H}_{A}\left(N_{A}-2\right)-\omega \hat{\mathbf{1}}\right| \times \operatorname{det}\left|\mathcal{H}_{B}\left(N_{B}-2\right)-\omega \hat{\mathbf{1}}\right|\right\}
\end{aligned}
$$

where, for simplicity, the interplane hopping $t_{A B}$ was assumed to be the geometrical average between $t_{A}$ and $t_{B}$. Note that for a given energy $\omega$ (and $\mathbf{q}_{\|}$) there is only one value of $\cos \left(q_{\perp} N d\right)$ defined. This indicates that the off-diagonal propagators oscillate as a function of the number of cells $N_{c}$ with a single period. In this case the Fermi surface is deformed as a function of $N_{A}$ and $N_{B}$ but the number of periods is left unchanged. This can be understood through the size reduction of the Brillouin zone along the perpendicular direction $q_{z}$, folding the Fermi surface back into the zone when it crosses the boundaries in $q_{z}= \pm \frac{\pi}{N d}$.

\section{B. Deformation of the Fermi surface}

Here, given two materials $A$ and $B$, we shall look at the change of the Fermi surface for different thicknesses $N_{A}$ and $N_{B}$. Still assuming the tight-binding parameters used in the evaluation of figure 2, the cross sections of the superlatticed spacer Fermi surface for $q_{x}=0$ are shown in figures 3 and 4 . Two cases are displayed, namely $N=3$ with $E_{F}=-1.6$ in figure 3 and $N=6$ with $E_{F}=-1.35$ in figure 1 , where $E_{F}$ represents the Fermi level. In all those figures the vertical axes are along the direction perpendicular to the layers whereas the horizontal direction represents $q_{y}$. The Fermi surfaces are drawn inside the first Brillouin zone represented by the rectangles, which are 
reduced along the $z$ direction by a factor $N$. Note that figures 3A $\left(N_{A}=0\right)$ and $3 \mathrm{D}\left(N_{A}=3\right)$ correspond to the bulk Fermi surfaces of the pure materials $B$ and $A$, respectively. The choice of different Fermi levels for $N=3$ and $N=6$ results from the analysis of figure 2, using the criterion of selecting a Fermi level which crosses the most number of bands. In fact, in figure $2 \mathrm{~A} E_{F}=-1.6$ yields solutions for all possible values of $N_{A}$ and $N_{B}$ whereas in figure 2 $\mathrm{B}$ $E_{F}=-1.35$ intersects the curves $N_{A}=2, N_{A}=3$ and $N_{A}=4$.

As discussed in section [1], in the case where only one period exists in the propagators, the RKKY geometrical picture coincides with the more general stationary phase conditions of Eq.(7) for selecting the effective oscillation periods of the coupling. Due to the equivalence between $q_{x}$ and $q_{y}$, it is clear from figures 3 and 1 that all wave vectors $q_{\perp}$ satisfying the stationary phase conditions are located at the origin of the 2-dimensional Brillouin zone, i.e., $q_{x}=q_{y}=0$. Therefore, the oscillation period of the propagator $g_{0 N_{c}}$ is associated with the value of $q_{\perp}$ determined by the solution of Eq.(16) for $q_{x}=q_{y}=0$.

According to Eqs. (11) and (2), the coupling is a function of the propagators $g$ between the magnetic planes. In the case considered here, $g_{0 N_{c}}^{\alpha \alpha^{\prime}}$ and $g_{N_{c} 0}^{\alpha^{\prime} \alpha}$ are the propagators involved. We recall that in this case the coupling depends on the product $g_{0 N_{c}}^{\alpha \alpha^{\prime}} g_{N_{c} 0}^{\alpha^{\prime} \alpha}$ and bearing in mind that both propagators oscillate with the same periods, the coupling oscillates with an effective wave vector which is twice that for $g_{0} N_{c}$. The corresponding periods of the coupling are then given by $\Lambda\left(N_{A}, N_{B}\right)=\pi / q_{\perp}$. The periods depend on the intra-cell composition determined by $N_{A}$ and $N_{B}$ as can be seen by the change in caliper of the extremal wave vectors spanning the Fermi surface in the centre of the Brillouin zones. Following Eq.(16), the periods are displayed in Table 1 and the product $g_{N_{c} 0}^{\alpha \alpha^{\prime}} g_{0 N_{c}}^{\alpha^{\prime} \alpha}$ as a function of $N_{c}$ for different intracell parameters and for $\alpha=\alpha^{\prime}=1$ is shown in figure 5 . Note the agreement between the periods in figure 5 and the ones in Table 1 .

\section{DISCUSSION AND CONCLUSIONS}

The results presented above indicate that the oscillation periods of the interlayer coupling across superlatticed spacers are related to the shape of the Fermi surface when the coupling is investigated as a function of the number of cells of the superlattice. Therefore, a change in the composition of the superlattice enables one to deform the corresponding Fermi surface and consequently tune the oscillation periods of the coupling. Moreover, although the present analysis is not concerned with the strength of the oscillation periods, it should be mentioned that the change in intra-cell parameters not only alters the relevant wave vectors but also the respective curvatures of the Fermi surface. Bearing in mind the dependence of the oscillation amplitude on such curvatures, the contribution of periods may be enhanced by the variation of the parameters $N_{A}$ and $N_{B}$. Non-RKKY periods, for instance, which in some cases contribute weakly to the oscillations $\mathrm{l}$, , could become more relevant through the variation of those parameters.

It is hoped that the simple case treated here reflects what occurs in real systems, which may be confirmed by future experiments. Alloys that, under certain critical temperatures, present ordered phases may be used to test the veracity of the present claims. In the ordered phase, $\mathrm{Cu}-\mathrm{Zn}$ alloys, for instance, have a BCC structure consisting of interpenetrating simple cubic sublattices ${ }^{\mathrm{H}}$. In this case, cells formed by one plane of $\mathrm{Cu}$ and one plane of $\mathrm{Zn}$ are periodically repeated, corresponding to a possible realization of the system proposed in this communication. The lattice parameters of the ordered $\mathrm{Cu}-\mathrm{Zn}$ alloy and that of $\mathrm{Fe}$ are similar, which may allow them to be grown in layered structures. Although the oscillation periods of the coupling cannot be tuned in this system, it is suggested that the periodicities associated with the corresponding Fermi surface will become evident only when plotted as a function of even (or odd) number of spacer layers. Alternatively, by growing superlattices of non-magnetic metals, e.g. noble metals, in wedge-shaped systems 19 , 19 one can change the intracell composition of the system to test whether the periods of the coupling can be tuned, as the present calculations indicate.

\section{ACKNOWLEDGEMENTS}

I wish to acknowledge Professor D. M. Edwards, Dr. R. B. Muniz, Dr. J. d'Albuquerque e Castro and Dr. R. Wright for their invaluable contributions to this work. Also, I am grateful to CNPq of Brazil for financial support. 


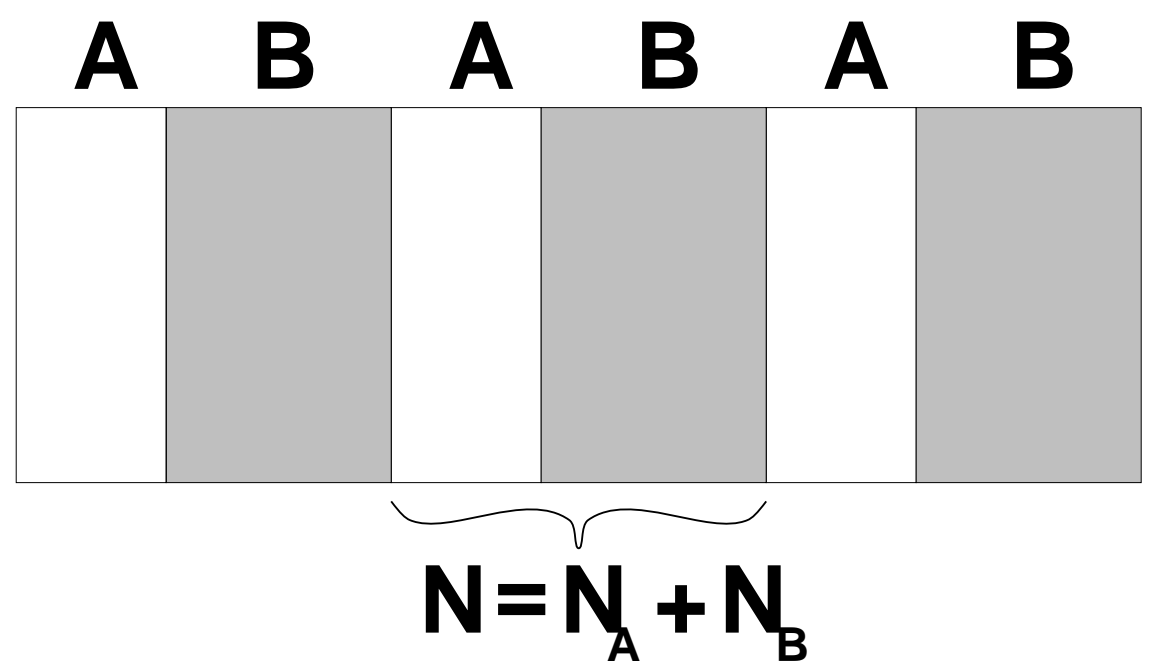

FIG. 1. Schematic representation of a superlatticed spacer. 
(A)

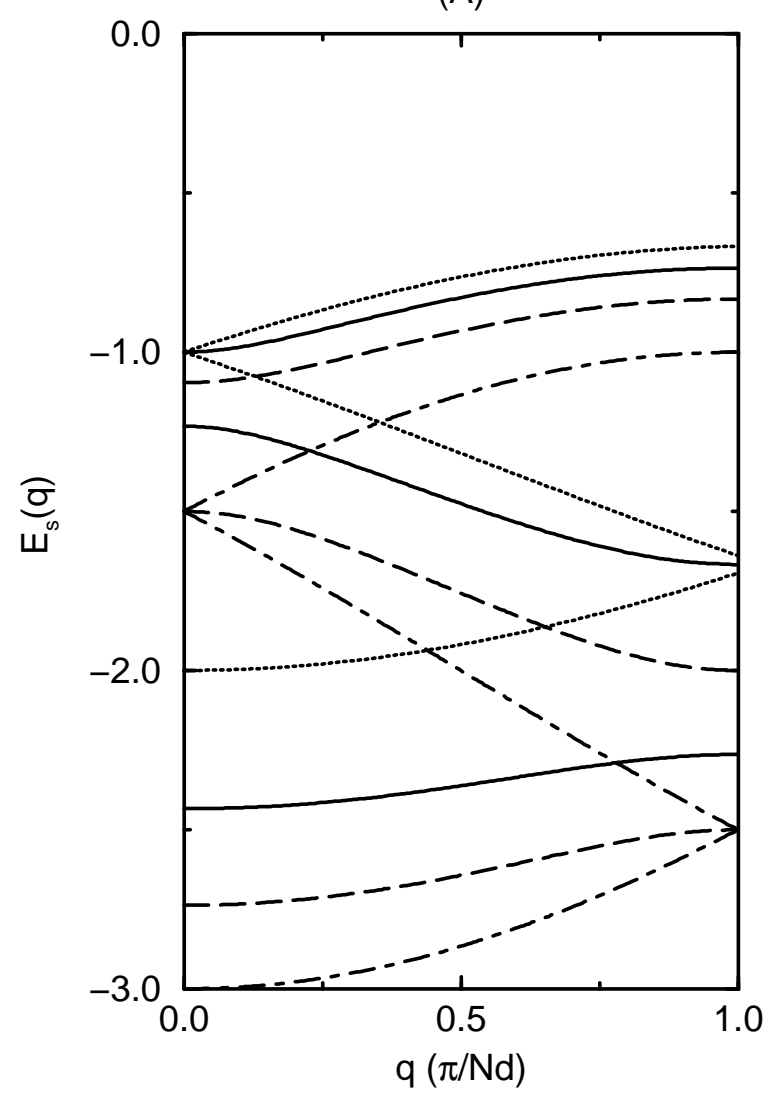

(B)

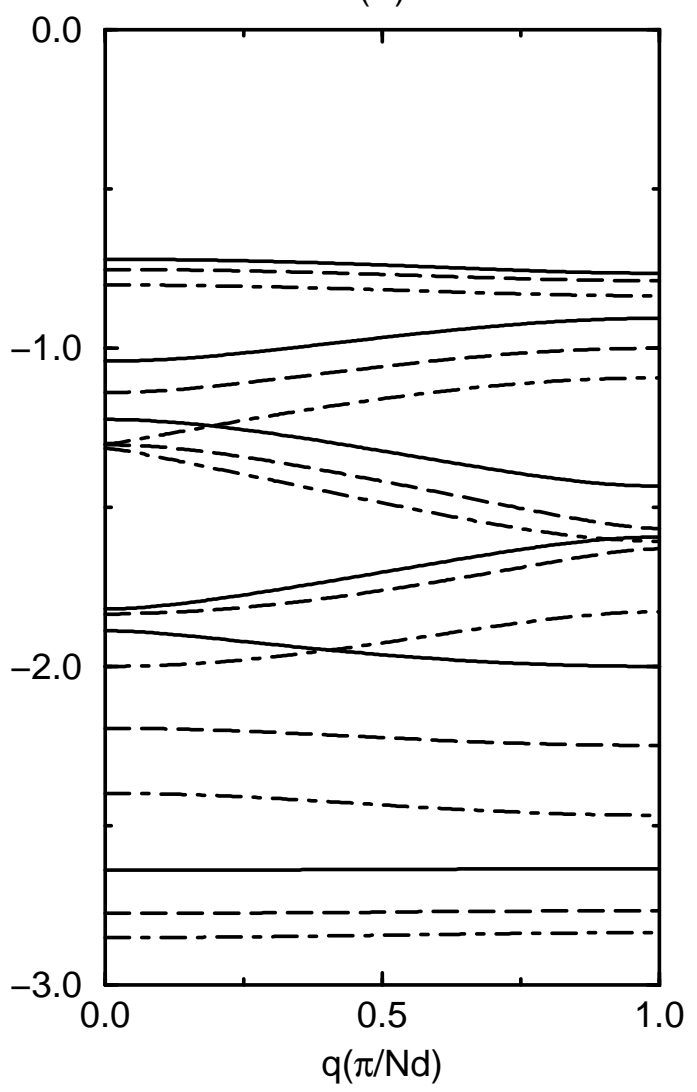

FIG. 2. Band structures $E_{s}\left(q_{\perp}, q_{x}=q_{y}=0\right)$ for (A) $N=3$ and (B) $N=6$ as a function of $q_{\perp}$. In figure A the dotted line is $N_{A}=0$, the full line is $N_{A}=1$, the dashed line is $N_{A}=2$ and the dot-dashed line is $N_{A}=3$. In figure B the the full line is $N_{A}=2$, the dashed line is $N_{A}=3$ and the dot-dashed line is $N_{A}=4$. 

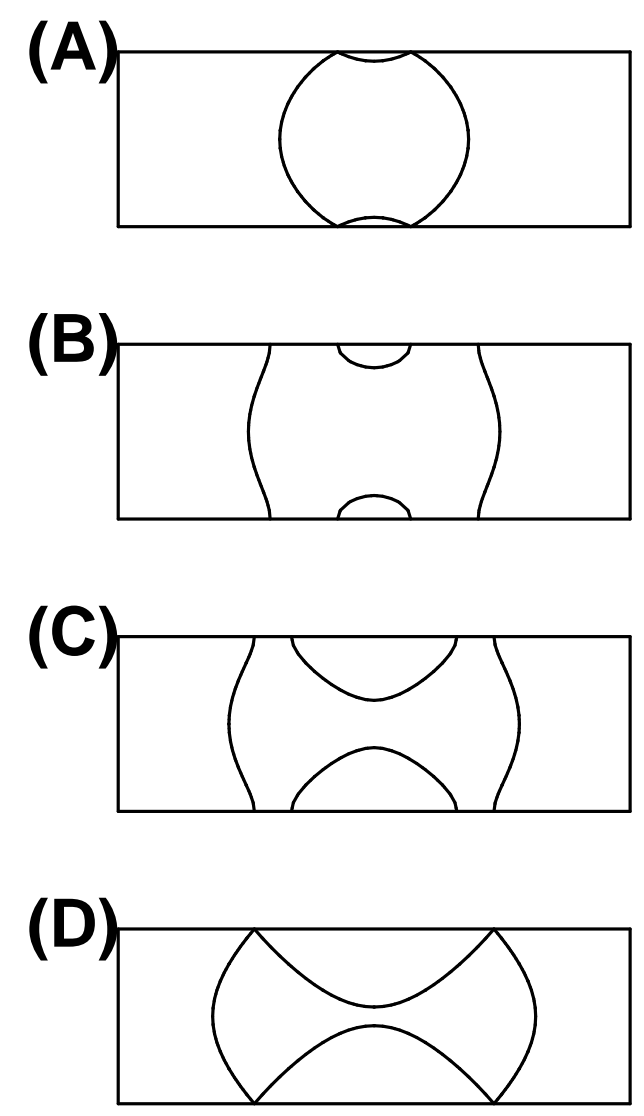

FIG. 3. Fermi surfaces for $N=3$. (A) $N_{A}=0$, (B) $N_{A}=1$, (C) $N_{A}=2$ and (D) $N_{A}=3$. Note that figures (A) and (D) correspond to the bulk Fermi surface of the single-material spacers $B$ and $A$, respectively. The rectangles are the Brillouin zones, whose dimensions are $2 \pi / d$ horizontally and $2 \pi / 3 d$ vertically. 

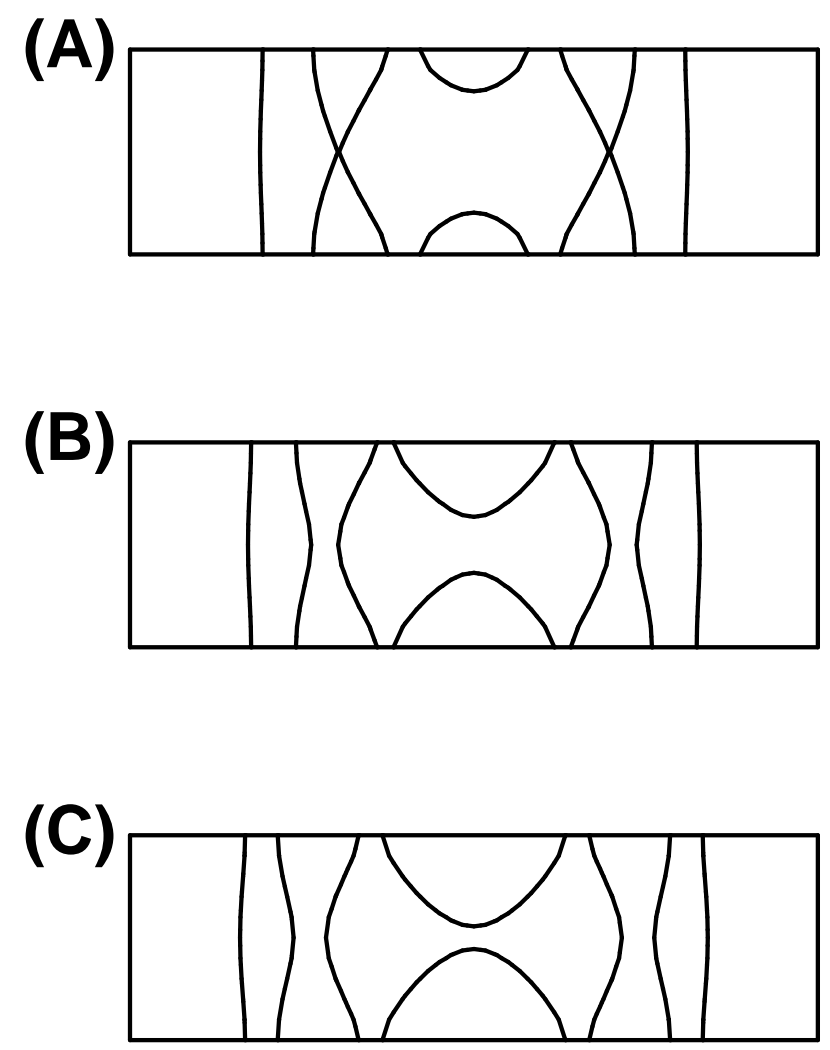

FIG. 4. Fermi surfaces for $N=6$. (A) $N_{A}=2$, (B) $N_{A}=3$ and (C) $N_{A}=4$. The rectangles are the Brillouin zones, whose dimensions are $2 \pi / d$ horizontally and $2 \pi / 6 d$ vertically. 

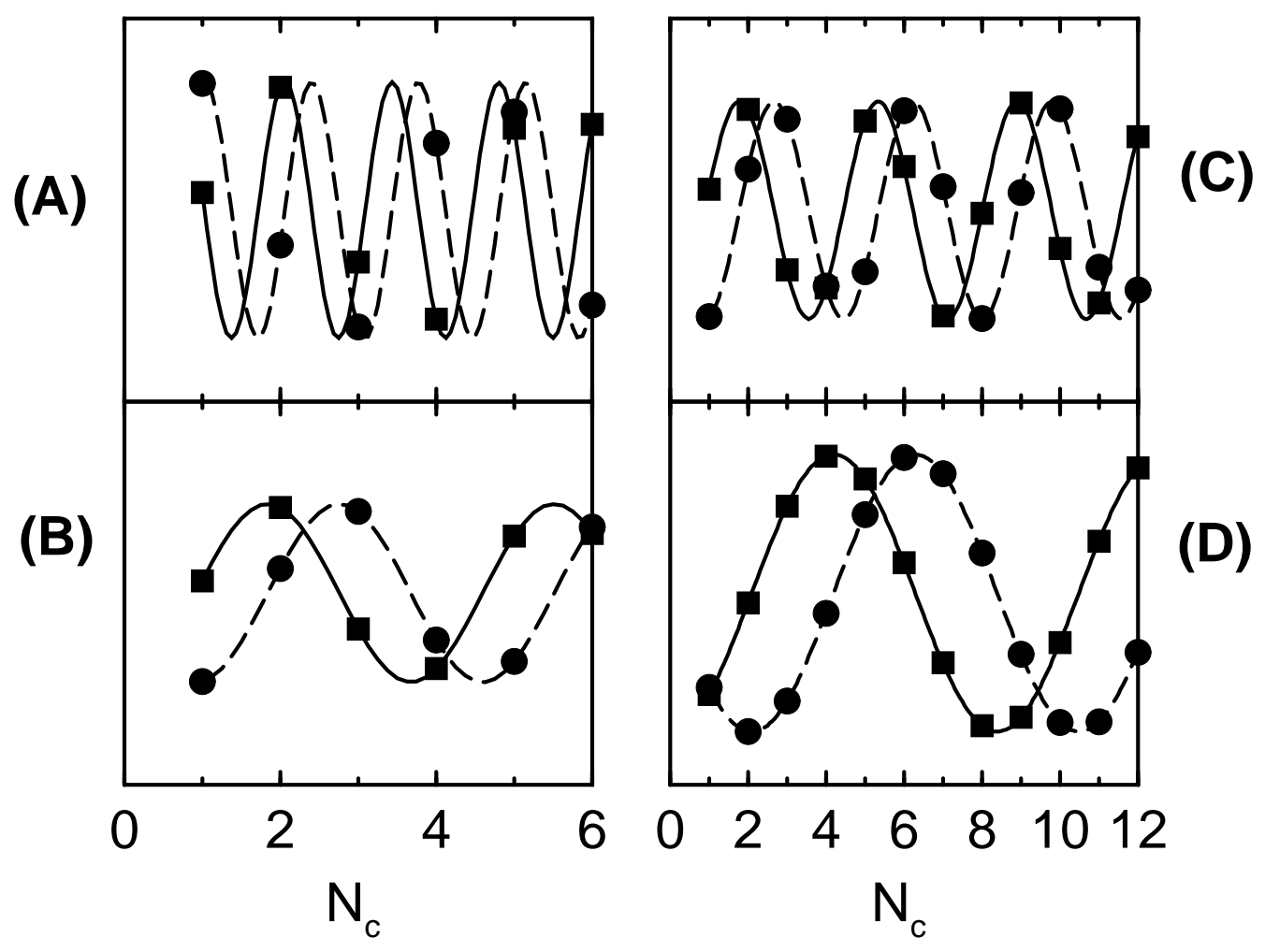

FIG. 5. $g_{N_{c} 0}^{\alpha \alpha^{\prime}} g_{0 N_{c}}^{\alpha^{\prime} \alpha}$ (in arbitrary units) as a function of the number of cells $N_{c}$ for some intracell parameters. $\alpha=\alpha^{\prime}=1$. The full lines with squares are the real part of the functions whereas the dashed lines with circles are the imaginary part. The symbols represent the integer values of $N_{c}$, whereas the lines are a guide to the eye. (A) $N_{A}=1$ and $N_{B}=2 ;(\mathrm{B}) N_{A}=2$ and $N_{B}=1 ;(\mathrm{C}) N_{A}=3$ and $N_{B}=3$; (D) $N_{A}=4$ and $N_{B}=2$. In cases (A) and (B) the Fermi level is $E_{F}=-1.6$ whereas $E_{F}=-1.35$ in cases (C) and (D). 


\begin{tabular}{lr}
\hline \hline$N=3$ & $N=6$ \\
\hline$\Lambda(0,3)=1.13$ & $\Lambda(2,4)=1.69$ \\
$\Lambda(1,2)=1.37$ & $\Lambda(3,3)=3.56$ \\
$\Lambda(2,1)=3.67$ & $\Lambda(4,2)=8.39$ \\
$\Lambda(3,0)=9.33$ & \\
\hline \hline
\end{tabular}

TABLE I. Oscillation periods $\Lambda\left(N_{A}, N_{B}\right)$ (in units of $D$ ) of the coupling as a function of the number of cells $N_{c}$ for different values of $N_{A}$ and $N_{B}$.

${ }^{1}$ B. Heinrich and J. F. Cochran, Adv. Phys. 42523 (1993)

2 B. Heinrich and J. A. C. Bland, Ultrathin Magnetic Structures - Vol.2 (1994)

${ }^{3}$ S. S. Parkin, N. More and K. P. Roche, Phys. Rev. Lett. 64, 2304 (1990)

${ }^{4}$ D. M. Edwards, J. Mathon, R. B. Muniz and M. S. Phan, Phys. Rev. Lett. 67, 493 (1991).

${ }^{5}$ P. Bruno and C. Chappert, Phys. Rev. Lett. 67, 1602 (1991)

${ }^{6}$ P. Bruno and C. Chappert, Phys. Rev. B 46, 261 (1992)

${ }^{7}$ D. M. Edwards, J. Mathon and R. B. Muniz, Phys. Rev. B 5016066 (1994)

${ }^{8}$ M. S. Ferreira, J. d'Albuquerque e Castro, D. M. Edwards and J. Mathon, J. Magn. Magn. Mater. 154, L1 (1996)

${ }^{9}$ M. S. Ferreira, J. d'Albuquerque e Castro, D. M. Edwards and J. Mathon, J. Phys.: Condens. Matter 8, 11259 (1996)

${ }^{10}$ A. T. Costa Jr, R. B. Muniz, J. d'Albuquerque e Castro, M. S. Ferreira and J. Mathon, Phys. Rev. B 55, 3724 (1997)

${ }^{11}$ J. d'Albuquerque e Castro, M. S. Ferreira and R. B. Muniz, Phys. Rev. B 49, 16062 (1994)

${ }^{12}$ S. N. Okuno and K. Inomata, Phys. Rev. Lett. 70, 1711 (1993)

13 J. d'Albuquerque e Castro, J. Mathon, Murielle Villeret and D. M. Edwards, Phys. Rev. B 51, 12876 (1995)

14 J. d'Albuquerque e Castro, unpublished.

15 J. d'Albuquerque e Castro, J. Mathon, Murielle Villeret and A. Umerski, Phys. Rev. B 53, 13305 (1996)

${ }^{16}$ R. A. Brualdi, Combinatorial Matrix Theory (1991)

${ }^{17}$ C. Kittel, Introduction to Solid State Physics, 7th Ed., (1996)

${ }^{18}$ I. Goldfarb, E. Zolotoyabko and D. Shechtman, J. Appl. Phys. 74, 2501 (1993)

${ }^{19}$ F. G. Aliev, E. Kunnen, K. Temst, K. Mae, G. Verbanck, V. V. Moshchalkov and Y. Bruynseraede, Phys. Rev. Lett. 78, $134(1997)$ 